\title{
Cross-Layer Software-Defined 5G Network
}

\author{
Mao Yang · Yong Li • Long Hu • Bo Li · Depeng Jin · Sheng Chen • \\ Zhongjiang Yan
}

Received: date / Accepted: date

\begin{abstract}
In the past few decades, the world has witnessed a rapid growth in mobile communication and reaped great benefits from it. Even though the fourth generation $(4 \mathrm{G})$ mobile communication system is just being deployed worldwide, proliferating mobile demands call for newer wireless communication technologies with even better performance. Consequently, the fifth generation $(5 \mathrm{G})$ system is already emerging in the research field. However, simply evolving the current mobile networks can hardly meet such great expectations, because over the years the infrastructures have generally become ossified, closed, and vertically constructed. Aiming to
\end{abstract}

This work is supported by National Basic Research Program of China (973 Program Grant No. 2013CB329105), National Natural Science Foundation of China (Grants No. 61301080, No. 61171065, 61271279, and 61201157), Chinese National Major Scientific and Technological Specialized Project (No. 2013ZX03002001 and 2014AA01A707), Chinas Next Generation Internet (No. CNGI-12-03-007), and the Fundamental Research Foundation of NPU (Grant No. JCY20130131).

M. Yang · B. Li · Z. Yan

School of Electronics and Information, Northwestern Polytechnical University, Xi'an 710072, P. R. China

E-mail: yangmao@nwpu.edu.cn

Y. Li · D. Jin

Department of Electronic Engineering, Tsinghua University, Beijing 100084, P. R. China

E-mail: liyong07@tsinghua.edu.cn

L. $\mathrm{Hu}$

School of Computer Science and Technology, Huazhong University of Science and Technology

E-mail: minchen.cs@gmail.com

S. Chen

Electronics and Computer Science, University of Southampton, Southampton SO17 1BJ, U.K.

E-mail: sqc@ecs.soton.ac.uk establish a new paradigm for $5 \mathrm{G}$ mobile networks, in this article, we propose a cross-layer software-defined 5G network architecture. By jointly considering both the network layer and the physical layer together, we establish the two software-defined programmable components, the control plane and the cloud computing pool, which enable an effective control of the mobile network from the global perspective and benefit technological innovations. Specifically, by the cross-layer design for software-defining, the logically centralized and programmable control plane abstracts the control functions from the network layer down to the physical layer, through which we achieve the fine-grained controlling of mobile network, while the cloud computing pool provides powerful computing capability to implement the baseband data processing of multiple heterogeneous networks. The flexible programmability feature of our architecture makes it convenient to deploy cross-layer technological innovations and benefits the network evolution. We discuss the main challenges of our architecture, including the fine-grained control strategies, network virtualization, and programmability. The architecture significantly benefits the convergence towards heterogeneous networks and it enables much more controllable, programmable and evolvable mobile networks. Qualitative simulations validate these performance advantages.

Keywords Software defined network - Network virtualization · Network Architecture · 5G

\section{Introduction}

With the rapid growth of mobile demands and the everincreasing use of smart phones, mobile network has been one of the fastest growing technologies that impact major aspects of our life [1]. In recent years, the $4 \mathrm{G}$ 
mobile communication system is being deployed worldwide, leading to a rapid growth in the mobile network capacity, which further dramatically stimulates the mobile demands. As mobile network evolves from voiceoriented to media-oriented [2,3], incremental improvements of networks can no longer keep the pace with the increase in mobile data demands, since there exist several challenges that the current mobile network can hardly address.

1) Convergence of heterogeneous wireless networks. There exist various heterogeneous wireless networks, i.e. pluralistic standards such as GSM, UMTS, LTE and WLAN, and they will coexist for a long time [4. However, as current wireless operators typically deploy their networks by vertically constructed method, these heterogeneous networks can hardly interconnect with each other, which makes operators incapable of efficiently optimizing and dynamically scheduling from the global perspective. For example, although there may be many mobile networks around us, we may not be able to access the most appropriate one or select multiple networks simultaneously. The only thing we can do is to access one specific network all the time, even if this network performs quite poorly.

2) Efficient utilization of wireless resources. As a result of the difficulty in achieving the convergence of heterogeneous networks, many network devices are not fully utilized and plenty of wireless resources are wasted, while generating enormous amounts of energy consumption. While several big carriers, including AT\&T, Verizon, T-Mobile and Sprint, say that in the next few years they may not have enough spectrum to meet the demands for mobile data, many scientists and engineers, including Martin Cooper, father of the mobile phone, are convinced that the main reason for spectrum crisis is that the spectrum resources are not fully utilized [5].

3) Network innovations. Proliferating mobile demands compel wireless operators to build sustained innovations to continuously boost their network performances. However, tightly coupling with specific hardware and lack of flexible control interfaces, the current mobile network can hardly provide a fast track for technological innovations.

4) Wireless applications and services. Wireless services proliferate significantly, e.g. Big Data [6,7, and different kinds of services require very different network characteristics. Unfortunately, the current mobile network often can only support these wide-ranging and very different services with the same network characteristics and, consequently, it may provide users with poor quality of service (QoS) and poor quality of experience $(\mathrm{QoE})$.
Because the current mobile network is vertically constructed over the years, it generally becomes ossified and closed, and has difficulty to meet the above mentioned challenges. This calls for the research and deployment of new generation of mobile network, specifically, $5 \mathrm{G}$, which is capable of providing a more open and efficient mobile network. In particular, $5 \mathrm{G}$ should benefit from the convergence of heterogeneous networks, facilitate network evolution, boost the network performance, enhance users' QoS and QoE, and at the same time save resources and energy. These objectives require mobile networks to offer much more efficient controlling and user data processing functions across the network layer down to physical layer by jointly considering the both layers together. However, since the physical layer technologies in mobile networks are much more complicated than in the wired scenarios, implementing efficient control and programmable user planes across the network layer and the physical layer is extremely challenging. The problem gets worse as the mobile environment becomes more complex, e.g. high mobility, frequent handovers, and heavy interferences. Consequently, the key technologies of $5 \mathrm{G}$ system are still open and attract increasingly attentions [8].

Software defined network (SDN), an innovative paradigm, advocates separating the control plane and data plane, and abstracting the control functions of the network into a logically centralized control plane, referred to as SDN controller [9, 10]. Aiming to establish a new paradigm for $5 \mathrm{G}$ mobile network, in this article, we propose a cross-layer software-defined $5 \mathrm{G}$ network architecture. In our architecture, the concept of SDN controller is extended to take the control functions of the physical layer into consideration as well, not just those of the network layer. Furthermore, we also introduce the novel cloud computing pool which considers the programmable user data processing functions of both the network layer and the physical layer jointly. Specifically, by abstracting the control functions of the both layers, we introduce a logically centralized programmable control plane oriented towards both network and physical layers, through which we achieve the fine-grained controlling and flexible programmability of the both layers. For example, we can control the packets forwarding in network layer as well as direct the beamforming and interference canceling in physical layer. The cloud computing pool proposed in our architecture provides powerful computing capability to implement the baseband data processing of multiple heterogeneous networks, which efficiently improves the convergence of heterogeneous wireless networks. Moreover, the programmable scheme in our architecture flexibly supports the network evolution and the deployment of 
any potential technological innovations which is particularly important as the key technologies of network and physical layers of $5 \mathrm{G}$. We also discuss the main technical challenges of our architecture, including the fine-grained control strategies oriented to the both network and physical layers, network virtualization that supports the customizability, and programmability that is beneficial to network evolution. Qualitative simulations are deployed to validate the advantages of our proposal. Our study demonstrates that our proposed $5 \mathrm{G}$ architecture is capable of achieving its design goals, namely, convergence of heterogeneous networks, finegrained controllability, efficient programmability for network evolution, and network and service customizability, and therefore it offers an architecture design of future mobile network.

The remainder of this article is organized as follows. We first offer a rethinking of mobile network, and this is followed by the introduction to SDN. Next, we present our cross-layer software-defined 5G architecture and analyze its features and system feasibility. Then we interpret the key technologies and challenges of our architecture. Several quantitative performance results are provided. Finally, we conclude the article by summarizing our contribution.

\section{Rethinking the Mobile Networking}

The challenges to the current mobile network are mostly caused by the ossification, closeness, and vertically constructed features. Simply evolving the traditional networking architecture can hardly meet the requirements of future mobile networks. We rethink the problems of the current mobile networking and envisage how the future mobile network looks like, and consequently propose the design goals of our architecture.

Ossified vs Controllable. Controlling in the current mobile network is limited and ossified. It is quite difficult to dynamically adjust the control strategies when the network status changes quickly. For example, many users with high mobility may go through numerous and frequent handovers, especially when the handovers occur among heterogeneous networks, which needs more fine-grained and dynamical controlling. Therefore, efficiently controlling is a fundamental function that the future network has to provide.

Closed vs Programmable. Technological innovations in mobile network need to be continuously developed in order to meet the demands. Unfortunately, mobile network technologies are usually solidified in the hardware. For example, the unequal error protection (UEP), a physical layer technology, can provide higher video quality while consuming less spectrum. But to make such performance enhancing changes typically require replacing the hardware due to the tightly coupling with the hardware in the form of specific ASICs designed for each protocol of the LTE network [11. In particular, dynamically deploying specific technologies based on the run-time network status becomes especially difficult. Therefore, it is necessary and highly desired to introduce greater programmability into mobile networks.

Vertical vs Converging. The vertical-constructed method has dominated the mobile networking almost since the first generation mobile communication system was conceived, and it has brought great successes to mobile communication. However, as the scale of mobile network is quickly enlarging and the network becomes ever-increasingly dense, traditional vertical-constructed networking severely restricts the development of mobile networks. For example, 3G, LTE and WLAN can hardly interconnect, and the operators are incapable of controlling the network from the global perspective. Thus, enabling efficiently convergence of heterogeneous networks must be supported in the future mobile network.

Unified vs Evolvable. Multiple mobile protocols and pluralistic standards will coexist for a long time [4. Each of them possesses the distinctive advantage for specific services or situations. For example, $3 \mathrm{G}$ provides better voice services and WLAN suits for video applications. The diversified world stimulates pluralistic demands, and thus it is almost impossible to find one unified network with solidified technologies to fullfill all the expectations. Consequently, the future mobile network must support smooth evolution for pluralistic standards.

Based on the above analysis, we can envisage the basic characteristics of the future mobile network and naturally define the design goals of our proposed $5 \mathrm{G}$ architecture, which are summarized as follows.

- Beneficial to the convergence of heterogeneous networks;

- Fine-grained controllability for mobile networks;

- High programmability and supply open interfaces for network technological innovations;

- High evolvability and fast deployment;

- Customizability of networks and services.

\section{Software Defined Network}

SDN has become one of the hottest topics in computer networking. By separating the control plane and the data plane, SDN abstracts the control functions of the network layer into a logically centralized control plane, 
known as the SDN controller [9,10. This SDN controller makes the rules and control the behaviors of data plane devices from the global perspective. OpenFlow [12 is a most common realization of SDN. Many manufacturers, such as HP, NEC, IBM, produce the commercial OpenFlow switch, and several kinds OpenFlow controllers, e.g. NOX and floodlight, are available. The OpenFlow protocol is sustainably released by Open Networking Foundation (ONF), and SDN is becoming ever-increasingly popular in networking today.

In recent years, several researchers attempt to extend SDN into wireless and mobile networks. OpenRadio 11 mostly focuses on the programmable data plane design of software-defined wireless network, but this work does not propose the holistic network structure with the control plane architecture. Li et al. 13 present a software-defined cellular network architecture, CellSDN, that covers both access network and core network. However, this work is oriented to the LTE network, and it can hardly be adapted to the circumstances of heterogeneous networks. SoftRAN 14 is a software-defined control plane of the radio access network by introducing a virtual big-base station, but this architecture mostly focuses on the one-tier situation, consisting of microcells, which is not suitable for the scenarios of heterogeneous networks. OpenRF [15] and SoftCell 16] focus on the software defined WiFi network and core network respectively. Meanwhile, MobiFlow [17] and OpenRoad [18. focus on the transport network that is similar to wired network and offer no special support for mobile access network. Moreover, almost all the existing works, except for OpenRadio and OpenRF, neglect the vital programmability features. Table 1 compares our proposed architecture with these existing works.

\section{Proposed 5G Architecture}

\subsection{Architecture Overview}

In order to address the challenges and to achieve the goals introduced in the previous sections, we propose a cross-layer software-defined 5G architecture, as depicted in Fig. 1, We introduce a logically centralized control plane, which makes the rules and controls the behaviors of network devices as well as provides appropriate application program interfaces (APIs) for various applications. To achieve the efficient controlling of both the network layer and physical layer, we design a fine-grained control strategy which is compatible with the both layers. Because the limitation of poor computing capability in baseband data processing and the lack of interconnection severely impede the traditionally designed physical layer's controllability, we introduce a

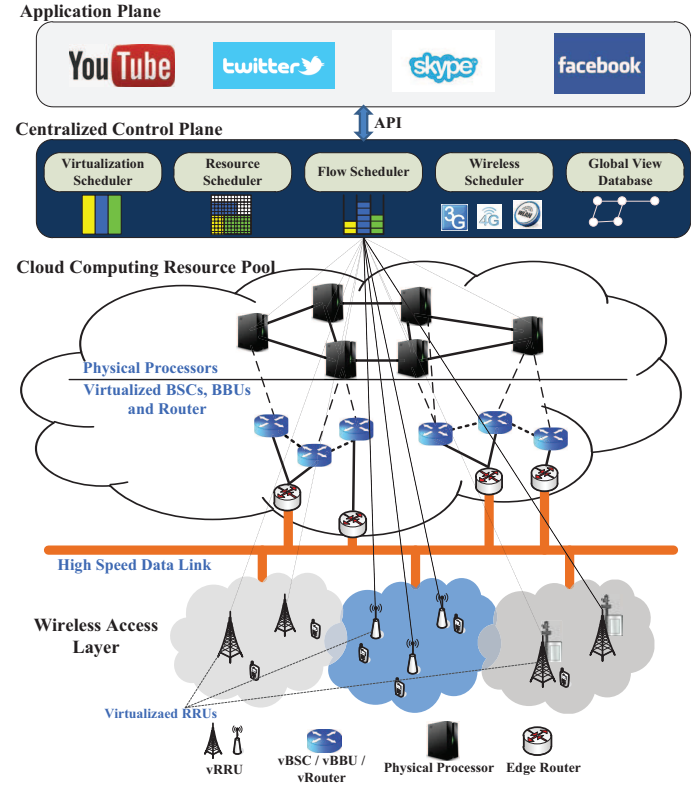

Fig. 1 Overview of our proposed 5G architecture.

novel cloud based baseband data processing pool. The radio transceiver devices and the baseband data processing devices are completely separated. We also introduce the programmability feature to make the network more evolvable and flexible for technological innovations. Our architecture contains four main parts: wireless access layer, cloud computing resource pool, centralized control plane, and application plane.

Wireless access layer consists of a large number of physical remote radio units (pRRUs) distributed at various locations. It enables several virtual RRUs (vRRUs) corresponding to different mobile communication protocols coexisting in one shared pRRU by radio frequency virtualization technology, to efficiently support fast deployment and the convergence of heterogeneous networks. For example, one pRRU can fast deploy one 3 GPP vRRU and one WLAN vRRU (vAP) simultaneously, according to the requirements.

Cloud computing resource pool is comprised of a large amount of physical processors with high-performance computing and storage capability as well as high speed links. The current mobile network adopts the vertically constructed networking, in which one RRU is tightly coupled with one specific base band unit (BBU). To loosen this inefficient coupling, in our architecture, the traditional BBUs, base station controllers (BSCs) and routers are replaced by virtual ones (vBBUs, vBSCs, and vRouters) which are implemented in the shared physical processors through virtualization. These shared physical processors can simultaneously run multiple vBBUs, vBSCs, and vRouters by allocating appropriate 
Table 1 Comparison between the existing works and our architecture.

\begin{tabular}{clll}
\hline & network architecture & physical layer controlling & programmability \\
\hline OpenRadio & $\sqrt{ } \times$ just data plane & $\times$ not involve & $\sqrt{ }$ \\
CellSDN & $\sqrt{ }$ & $\sqrt{ }$ & $\times$ not involve \\
SoftRAN & $\sqrt{ } \times$ but macro-cell situation & $\sqrt{ }$ & $\times$ not involve \\
OpenRF & $\times$ & $\sqrt{ }$ & $\times$ not involve \\
SoftCell & $\sqrt{ }$ & $\times$ not involve & $\times$ WiFi oriented \\
MobiFlow & $\sqrt{ }$ & $\times$ not involve & $\times$ core network \\
Our Architecture & $\sqrt{ }$ & $\sqrt{ }$ & $\times$ not involve \\
\hline
\end{tabular}

computing resources and storage resources. Specifically, each vBBU can deploy appropriate wireless protocol softwares to implement the physical layer programmability. The corresponding virtual elements (vBSCs, vBBUs, vRouters, and vRRUs) constitute a complete wireless network with specific protocols. Our cloud based data processing pool significantly improves the computing capacity of baseband processing and overcomes the interconnection difficulty of traditional mobile networks, which greatly benefits the convergence of heterogeneous networks. To guarantee the run-time properties, the wireless access pool is connected to the cloud computing resource pool through high speed optical links. The wireless access layer and the cloud computing resource pool comprise the data plane of our architecture.

The centralized control plane is the "brain" of our architecture by abstracting and combining the control functions of both the network and physical layers. The control plane can acquire the configurations and realtime status of the both layers to make the decision from the global perspective. We partition the control plane into several functional submodules.

1) Flow scheduler, which is related to the network layer, is similar to the wired SDN controller. It makes the polices and schedules the behaviors of each virtual forwarding element, i.e. guiding them to process the packets.

2) Wireless scheduler, which is oriented to the physical layer, is the "highlight" in our architecture. It can deploy appropriate wireless protocol softwares into each vBBU. Moreover, it also dynamically schedules the physical layer actions, for example, beamforming, power control, interference cancelling, massive MIMO and carrier aggregation.

3) Virtualization, which is an indispensable element of SDN, makes it possible to customize networks and services. Specifically, virtualization scheduler manages the strategies of virtualization. We introduce three kinds of virtualization - flow space virtualization, cloud level virtualization, and spectrum level virtualization - that conspicuously improve the network performance from different angles.

4) Resource scheduler. Because there are various resources in our architecture, including spectrum, computing, and storage, it is necessary to efficiently allocate these resources to different virtual elements, which is implemented in resource scheduler.

5) Global-view database. All the above-mentioned submodules need to know the static configurations and dynamic status of the whole network and, therefore we introduce a database with global view to collect these information.

The application plane contains a variety of network applications, each of which utilizes the APIs abstracted by the control plane to guarantee its QoS and QoE.

\subsection{Meeting the Design Goals}

We now emphasize that our proposed cross-layer softwaredefined 5G architecture achieves the design goals set out in Section 2] Firstly, we abandon the traditional vertically constructed networking method and introduce a cloud computing pool to process the baseband data of heterogeneous networks. Therefore, our architecture conspicuously benefits the convergence of heterogeneous networks. With our architecture, the mobile network can easily acquire real-time global network view, which enables its centralized control plane to efficiently control the entire network by considering both the network and physical layers as a whole. This makes the network much more open and controllable. The flexible programmability proposed not only provides the opportunities for fast deployment of technological innovations but also makes the network more evolvable. Our proposed three types of virtualization further enhance the customizability of the networks and services.

\subsection{Feasibility Analysis}

Recent years, SDN and network virtualization attract increasingly attentions from both the academic and in- 
dustrial communities. Many prototypes, testbeds and products have been developed and produced, in particular, OpenFlow controller, switch and protocols, as well as GENI network virtualization platform. Furthermore, data center network and cloud computing also stimulate fast development of SDN and virtualization technologies. Moreover, there are plenty of prototypes and testbeds for software defined radio (SDR) [19] as well as its extended version, cognitive radio [20, which offer considerable programmability. We also note that China Mobile Ltd. positively focuses on the research and development of the cloud based mobile network architecture, referred to as CRAN 21. Based on these progresses, we believe that our proposed architecture is quite feasible.

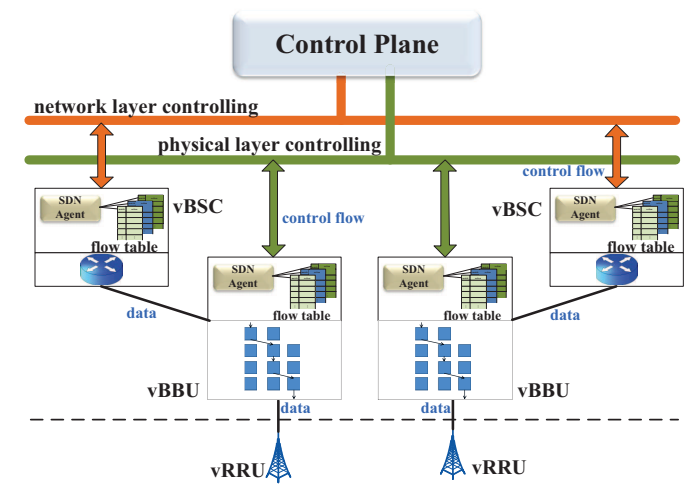

Fig. 2 The proposed fine-grained control strategy architecture.

\section{Key Challenges and Technologies}

\subsection{Fine-Grained Control Strategy}

The first technical challenge is to design the fine-grained control strategy, and we divide the problem into two subproblems: jointly optimizing strategy and individual control strategies.

The jointly optimizing strategy makes the decision to achieve the efficient controlling according to the static configurations and real-time status of both the network and physical layers. Consider for example that the QoS for a user at cell boundary deteriorates. We may need to determine the appropriate routing and bandwidth requirements as well as to schedule the beamforming and interference cancelling. After the decision has been made, the control plane decomposes the decision into two parts corresponding to the two layers, and then leverages the flow based "match-action" strategy for the both layers to make rules and control behaviors of each virtual element, as illustrated in Fig. 2. Correspondingly, each data plane device integrates a SDN agent, which complies with the control strategy, resolves the control flow, and communicates with the control plane. Virtual elements constantly report the real-time status to the control plane, which may then dynamically refresh the global-view database.

Since the responsibilities of the network layer and physical layer are distinctly different, the individual control strategies for the two layers are different, which defines the second subproblem of our fine-grained control strategy.

Network Layer. Since the packet header of each flow possesses several function fields called match fields, such as IP address and MAC address, the data plane devices can process the arriving packets by checking the match fields. Specifically, when a virtual element receives a packet, it first checks whether this flow matches its control rules. If so, it executes the corresponding actions. Otherwise, the packets will be dropped or sent to the control plane. An example can be interpreted as: if dest $\mathrm{IP}=\mathrm{xx} \cdot \mathrm{xx} \cdot \mathrm{xx} \cdot \mathrm{xx}$, then forward to vBBU1 with speed $\mathrm{S} 1$.

Physical Layer. Although the physical layer controlling also adopts the "match-action" strategy, the match fields, rules, and actions are quite different from the network layer. Since key technologies of $5 \mathrm{G}$ physical layer are still open, the format of match fields should be elastic. For example, the basic information filed should contain the encoding mode, modulation type, carrier information, etc. Additionally, the power control field may present the power control information, while the beamforming field and the interference cancelling field should provide some corresponding information. The control plane dynamically makes the decision according to the run-time status, and then sends control flows to the vBBUs. The "actions" in vBBUs refers to the appropriate physical layer technologies. For example, when QoS of a user deteriorates, the control plane may launch beamforming, while when the users at cell boundaries encounter severe inter-cell interference, the control plane may start interference cancelling.

\subsection{Network Virtualization}

Network virtualization as a promising means of the future network enables multiple concurrent virtual networks to run on the shared substrate resources [22, 1. Network virtualization is an indispensable part of SDN, while SDN architecture facilitates network virtualization. Network virtualization is responsible for customizing the networks and services. Therefore, how the virtualization scheme is designed directly influences the 


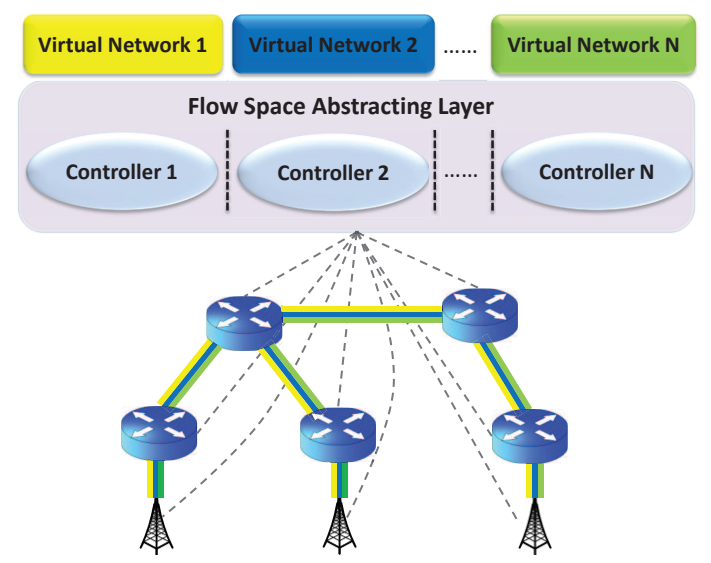

Fig. 3 Flow space virtualization for networks or services.

system performance and resource utilization. There are three types of virtualization, flow space virtualization, cloud level virtualization, and spectrum level virtualization, in our architecture, and we now elaborate each of them.

Flow space virtualization. Different operators or network services require different network characteristics. As the control strategy in our architecture is flow based, flow space can be divided into multiple slices, each of which corresponds to one virtual network. As shown in Fig. 3, we introduce a flow space abstracting layer. By abstracting the control functions of virtual elements, this layer slices the flow space according to the requirements of operators or services, and provides each flow slice with one controller. Then virtual networks are generated, and each virtual network is managed by its corresponding controller. The virtual networks share the same underlying data plane devices. In vBSC or vRouter, different virtual networks have different rules and run different actions. Operators often need different vBBUs due to the specific wireless protocols.

Cloud level virtualization. The control plane first creates vBBUs, vBSCs and vRouters by virtualizing physical processors and allocating appropriate computing and storage resources. Then it establishes the forwarding and data processing rules for virtual elements. Moreover, it deploys the corresponding wireless protocol softwares in vBBUs by utilizing the programmability.

Spectrum level virtualization. It refers to the virtualization of spectrum by radio frequency virtualization technology, which enables several vRRUs with different wireless protocols to coexist in one shared pRRU. Spectrum level virtualization extends the virtualization scope, lessens the networking difficulty and saves the cost.

\subsection{Programmability}

The physical layer programmability faces several challenges including how to guarantee sufficient computing capability and how to efficiently implement physical layer technologies. Our proposed cloud based baseband processing pool provides powerful computing capability. In the physical layer, although different wireless protocols operate quite different from each other, they always share some same modules, such as modulation, coding and interleaving. Inspired from SDR, we modularize wireless protocols and establish a wireless module library in vBBUs. vBBUs can select different modules, and then connect these selected modules to implement the specific protocol. As 5G physical layer technologies are still open, our proposed programmability provides the flexibility for technological innovations, benefits the future network evolution, as well as enables fast deployment and customization of wireless protocols.

\section{Performance Evaluation}

Although in the current stage the architecture proposed has not been deployed in a real scenario, we try to evaluate the performance with real traces from a large cellular deployment. We show that our proposed architecture significantly improves the energy efficiency and QoS utility.

\subsection{Dataset}

To truly reflect the performance advantages, we use the real datasets named "Sitefinder" which is released by Ofcom on behalf of UK's government 23. These datasets record massive base stations information supplied by the mobile network operators. The information contains location information (latitude and longitude), operator information, base station type, transmission power, communication protocol (GSM, UMTS, and TETRA), frequency band, antenna information, and etc. Considering that the geographical area covered by these datasets is too wide, we select $10 \mathrm{~km} \times 10 \mathrm{~km}$ geographic area from Manchester. Consequently, there are 159 GSM base stations as well as 255 UMTS base stations deployed by five operators i.e. O2, Orange, Three, T-Mobile, and Vodafone.

\subsection{Energy Efficiency}

In recent years, many researchers focus on the energy efficiency in mobile communications and, as a result, 
lots of energy-efficient mechanisms are presented. However, a form of stalemate exists: these efficient mechanisms are difficult to implement and deploy in real scenarios since the network functions are highly solidified in the hardware and the network devices are inflexible to control. Meanwhile, our proposed architecture provides flexible programmability and centralized controlling, which makes it easy to implement these energyefficient schemes in real scenarios.

Considering that network traffic at midnight is low, i.e. tide effect, we propose two energy-efficient strategies: 1) the centralized control plane intelligently turn off the base stations on behalf of individual operator, referred to as IIT strategy; 2) control plane turn off the base stations from global perspective, i.e. cooperation cross operators, referred to as CIT strategy. It is notable that both of these two strategies needs to guarantee the whole area coverage. Fig. 4 depict the energysaving performance of GSM and UMTS. As coverage range of each base station increases, both strategies turn more base stations and save more energy. For example, when coverage range is $1000 \mathrm{~m}, 39$ percent base stations can be turned off and 35 percent energy can be save by utilizing IIT strategy for UMTS. Moreover, as figures shown, apparently, the CIT strategy achieves much more energy-efficient benefits than IIT since CIT allows the control plane to optimize the energy-efficient solution across multiple mobile operators.

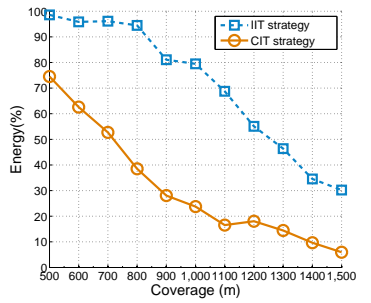

(a) Energy Ratio for GSM

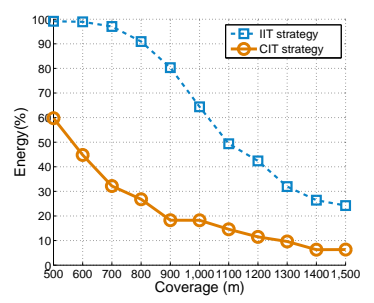

(c) Energy Ratio for UMTS (d) Base station Number for UMTS

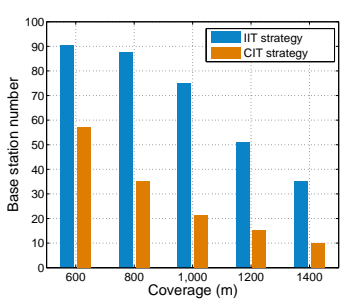

(b) Base station Number for GSM

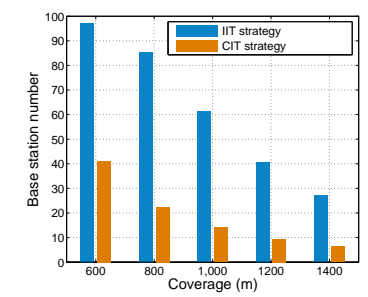

(d) Base station Number rar
6.3 QoS utility

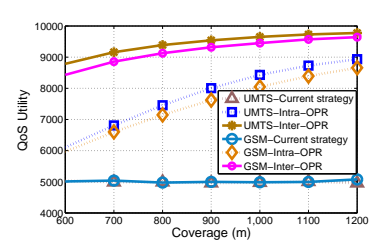

(a) Strategy 1-3

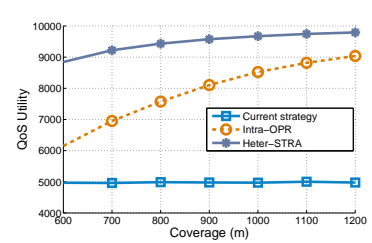

(b) Strategy 4
Fig. 5 QoS utility comparison.

In the current mobile network, every end user has no choice but to access one specific network. They may not be able to access the most appropriate one or select multiple networks simultaneously, which leads to poor QoS. In contrast, in our architecture, all the network devices are programmable and controlled by the centralized control plane. Consequently, the control plane may guide the end users to access the most appropriate networks, by which QoS can be improved.

We assume that there are 10,000 end users and each one runs an ongoing mobile service. We define the QoS utility between each pair of <end user, base station>, and assume the utility follows the uniform distribution in the range of $[0,1]$ by normalizing. The location (latitude and longitude) of each end user is randomly generated. We introduce four types of strategies: 1) Current strategy. Each business is bound to one specific network. 2) Intra operator optimizing (Intra-OPR): the control plane guides each end user to access the base station, belonging to the specific operator, with the largest QoS utility. 3) Inter operator optimizing (InterOPR): breaking the limit of one specific operator. 4) Breaking the limit of operator as well as one communication standard (Heter-STRA), i.e. it allows the end users to the access the base station among several heterogeneous networks (in this senario: cross UMTS and GSM). As Fig. 5(a) shows, both Intra-OPR and InterOPR obtain much more QoS utility than current strategy, and this advantage becomes much more obvious as the coverage range increases. After that, we consider the heterogeneous scenario and combine $159+255=414$ base stations. Fig. 5(b) confirms the perform advantages of Heter-STRA.

\section{Summary and Conclusions}

In this article, we have proposed a cross-layer softwaredefined 5G architecture, which addresses some of the 
key technical challenges facing the future mobile network. Our architecture is able to control the mobile network from the global perspective through the finegrained controlling for the both network and physical layers. Moreover, the cloud computing pool in our architecture provides powerful computing capability to implement the baseband data processing of multiple heterogeneous networks, while the programmability feature efficiently supports the network evolution and technological innovations.

The key technologies we introduced to our architecture include fine-grained control strategy, network virtualization, and programmability, and the key features of our proposed 5G architecture are controllability, programmability, evolvability and customizability. Since the key technologies of 5G are still open, we believe our flexible architecture is capable of supporting the $5 \mathrm{G}$ standards that eventually emerge and efficiently satisfying the requirements of mobile demands.

\section{References}

1. Zaki Y, Zhao L, Goerg C, Timm-Giel A (2011) Lte mobile network virtualization. Mob Netw Appl 16(4):424-432

2. Karjaluoto $H$ (2007) An investigation of third generation $(3 \mathrm{~g})$ mobile technologies and services. Contemporary Management Research 2(2):91-104

3. Kennedy M, Ksentini A, Hadjadj-Aoul Y, Muntean G (2013) Adaptive energy optimization in multimediacentric wireless devices: A survey. IEEE Commun Surv Tutor 15(2):768-786

4. Ericsson (2012) Mobile report on the pulse of the networked society. Ericsson Mobility Report. http://www.ericsson.com/res/docs/2012/ericsson-mobil Accessed november 2012

5. Chen B X. (2012) Carriers warn of crisis in mobile spectrum. The New York Times.

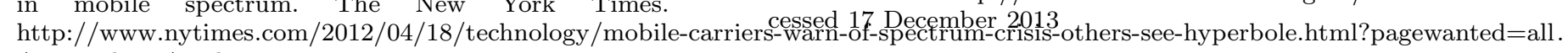
Accessed 17 April 2012

6. Chen M, Mao S, Liu Y (2014) Big data: a survey. Mob Netw Appl 19(2):171-209.

7. Chen M, Mao S, Zhang Y, Leung V (2014) Big data: related technologies, challenges and future prospects. SpringerBriefs in Computer Science.

8. Wang X, Chen M, Taleb T, Ksentini A, Leung V. (2014) Cache in the air: enabling the green multimedia caching and delivery for the $5 \mathrm{G}$ network. IEEE Commun Mag 52(2):131-139

9. Yu M, Rexford J, Freedman M J, Wang J (2010) Scalable flow-based networking with difane. SIGCOMM Comput Commun Rev 41(4):351-362

10. Kim H, Feamster N (2013) Improving network management with software defined networking. IEEE Commun Mag 51(2):114-119

11. Bansal M, Mehlman J, Katti S, Levis P (2012) Openradio: a programmable wireless dataplane. In: Proceedings of the first workshop on Hot topics in software defined networks, ser. HotSDN '12, New York, USA, pp 109-114

12. McKeown N, Anderson T, Balakrishnan H, Parulkar G, Peterson L, Rexford J, Shenker S, Turner J (2008)
Openflow: enabling innovation in campus networks. SIGCOMM Comput Commun Rev 38(2):69-74

13. Li l, Mao Z, Rexford J (2012) Toward software-defined cellular networks. In: 2012 European Workshop on Software Defined Networking (EWSDN), Berlin, Germany, pp 7-12

14. Gudipati A, Perry D, Li L E, Katti S (2013) Softran: software defined radio access network. In: Proceedings of the second ACM SIGCOMM workshop on Hot topics in software defined networking, ser. HotSDN '13, New York, USA, pp 25-30

15. Kumar S, Cifuentes D, Gollakota S, Katabi D (2013) Bringing cross-layer mimo to today's wireless lans. SIGCOMM Comput Commun Rev 43(4):387-398

16. Jin X, Li L E, Vanbever L, Rexford J (2013) Softcell: Scalable and flexible cellular core network architecture. In: Proceedings of the Ninth ACM Conference on Emerging Networking Experiments and Technologies, ser. CoNEXT '13, New York, USA, pp 163-174

17. Pentikousis K, Wang Y, Hu W (2013) Mobileflow: Toward software-defined mobile networks. IEEE Commun Mag 51(7):44-53

18. Yap K K, Sherwood R, Kobayashi M, Huang T Y, Chan M, Handigol N, McKeown N, Parulkar g (2010) Blueprint for introducing innovation into wireless mobile networks. In: Proceedings of the Second ACM SIGCOMM Workshop on Virtualized Infrastructure Systems and Architectures, ser. VISA '10, New York, USA, pp 2532

19. Palkovic M, Raghavan P, Li M, Dejonghe A, L. Van der Perre, and Catthoor F (2010) Future software-defined radio platforms and mapping flows. IEEE Signal Processing Magazine 27(2):22-33

20. Sengupta S, Hong K, Chandramouli R, and Subbalakshmi K P (2011) SpiderRadio: a cognitive radio network with commodity hardware and open source software. IEEE Commun Mag 49(3):101-109

21. China Mobile Institute (2011) C-ran: The road towards green ran white paper. China

ity-report november-2012.pdy pa R (2010) A survey of networn viluatiuation. Computer Networks 54(5):862-876

23. Ofcom (2012) Sitefinder: Mobile Phone Base Station Database. http://www.sitefinder.ofcom.org.uk/ Ac- 